\title{
Investigação de Novas Estratégias para o Ensino de Bioquímica Estrutural por meio de Realidade Aumentada
}

\author{
Alan Ferreira Alves1, Cícero Francisco Bezerra Felipe1, Liliane S. Machado2 \\ 1Departamento de Biologia Molecular e 2 Departamento de Informática \\ Universidade Federal da Paraíba (UFPB) \\ Cidade Universitária s/n - João Pessoa/PB - 58051-900 - Brasil \\ xxalanferreira@gmail.com, cicero@dbm.ufpb.br, liliane@di.ufpb.br
}

\begin{abstract}
This study aimed to investigate the difficulties of the students that attend to the Structural Biochemistry classes and the use of the augmented reality (AR) technologies as a support to the learning process. The work was divided into three phases: 1) application of a quiz, identifying the main difficulties with the subject; 2) development of a prototype with augmented reality technology; 3) use of the prototype and application of a quiz on the benefits identified with an AR technology in the learning process. It was observed that the main difficulty of the students consisted in the spatial visualization of biomolecules and the creation of an AR-based app would bean important tool in the process of learning the content of Structural Biochemistry.
\end{abstract}

Resumo. Este estudo buscou investigar as dificuldades dos alunos da disciplina Bioquímica Estrutural e a utilização de tecnologias de realidade aumentada como apoio no processo de aprendizagem. O trabalho ocorreu em três fases: 1) aplicação de questionário identificando as dificuldades dos alunos com a disciplina; 2) desenvolvimento de um protótipo com tecnologia de realidade aumentada; 3) uso do protótipo e aplicação de questionário acerca dos beneficios identificados com a tecnologia de RA no processo de aprendizado. Verificou-se que uma das maiores dificuldades dos alunos consistiu na a visualização espacial de biomoléculas e que a criação de um aplicativo baseado em RA poderia ser uma ferramenta importante no processo de aprendizagem na disciplina em questão.

\section{Introdução}

Nos últimos anos, a educação superior vem passando por mudanças de paradigmas relacionadas ao processo ensino-aprendizagem. Neste contexto, o uso de metodologias tradicionais nas quais o aluno é um sujeito passivo de aprendizado, tem sido direcionado para um processo mais dinâmico de ensino, focado na figura do discente, por meio das metodologias ativas de aprendizagem (GARZÓN, 2014), que consistem em estratégias de ensino cujo objetivo é motivar a busca e reflexão do conhecimento (MITRE, 2008). Essa necessidade de novas estratégias no processo ensino-aprendizagem é uma realidade, principalmente no ensino universitário. Para tanto, um conjunto de habilidades e aptidões deve ser desenvolvido nos alunos com a finalidade de estimular o senso crítico, a dinâmica de comunicação e a capacidade para resolução de problemas (SOUZA, 2006). 
As inúmeras transformações tecnológicas que facilitam o acesso às informações têm modificado o padrão tradicional de educação e exigido novas posturas dos professores e alunos, sendo necessária uma técnica moderna de ensino para que a performance acadêmica dos estudantes nas áreas das ciências melhore (LIMA, 2017). Uma ferramenta que vem auxiliando no processo de aprendizagem são as Tecnologias da Informação e Comunicação (TICs), as quais segundo Tavares, Souza e Correia (2013), são definidas como "um conjunto de recursos tecnológicos que podem proporcionar comunicação e/ou automação de diversos tipos de processos em diversas áreas e principalmente no ensino e na pesquisa".

As TICs fundamentam-se principalmente na visão construtivista de Jean Piaget, que pressupõe a criação de ambientes de aprendizagem que possibilitam a construção de algo e relação ativa do aluno na construção e realização de tarefas. Dentre as principais vantagens que as novas Tecnologias da Informação e Comunicação (TICs) oferecem na elaboração de projetos e materiais didáticos são: a capacidade de interatividade, a possibilidade de interação a distância, e o armazenamento e organização de informações representadas de várias formas, tais como textos, vídeos, animações, jogos lúdicos e áudios.

Dentre as metodologias que se destacam pelo uso das TICs no processo ensinoaprendizagem, tem-se a realidade aumentada (RA), cujo objetivo consiste em integrar objetos tridimensionais ao mundo real. $\mathrm{Na} \mathrm{RA}$, o usuário pode interagir com objetos virtuais que são inseridos no mundo real, promovendo a possibilidade de visualizar, explorar e modificar esses objetos (TORI e HOUNSELL, 2018).

O processo ensino-aprendizagem das disciplinas de Bioquímica, no geral, é desafiador pela natureza do conteúdo, o qual possui uma terminologia ampla e específica (nomes, fórmulas e estruturas químicas), por exigir um alto grau de abstração por parte do aluno, associado à necessidade de um conhecimento prévio em Química (GARZÓN, 2014) e por conter um grande volume de informações, que devem ser restringidas para adequar-se ao tempo disponível dos componentes curriculares (YOKAICHIYA, 2004). A bioquímica fundamenta-se em mostrar em termos moleculares as estruturas, os mecanismos e os processos químicos compartilhados por todos os organismos (NELSON \& COX, 2002). O ensino da Bioquímica na graduação é considerado interdisciplinar, uma vez que se encontra presente na maioria dos cursos das áreas de saúde e biológicas, tais como: Farmácia, Fisioterapia, Biologia, Nutrição, dentre outros. Sua metodologia abrange um campo extenso de compreensão e interpretações que usa princípios presentes no ensino da Química e da Biologia, utilizando recursos que podem ser trabalhados na sua forma teórica e/ou prática (ALCÂNTARA, 2015).

O presente estudo tem como objetivo apresentar os resultados de uma pesquisa realizada com alunos acerca das dificuldades quanto ao aprendizado de bioquímica, bem como, os benefícios potenciais, por eles identificados, quanto ao uso da RA neste processo.

\section{Metodologia}

Para realização do estudo, três etapas foram definidas: 1) aplicação de questionário identificando as dificuldades dos alunos com o aprendizado de bioquímica; 2) desenvolvimento de um protótipo de visualização de molécula com RA; 3) uso do protótipo e aplicação de questionário acerca dos benefícios identificados com a tecnologia 
de RA no processo de aprendizado. Os dois questionários foram aplicados para os alunos de do curso superior de Farmácia matriculados na disciplina de Bioquímica Estrutural no período de março a junho de 2018. O primeiro questionário consistiu em seis perguntas abertas e fechadas e foi aplicado para um total de vinte e cinco alunos.

Os resultados obtidos com o primeiro questionário subsidiaram $\mathrm{o}$ desenvolvimento de um protótipo de aplicativo para dispositivos móveis baseado em realidade aumentada para visualização de uma estrutura molecular discutida na disciplina. Após a apresentação do protótipo e uso pelos alunos, um segundo questionário foi aplicado para capturar as impressões dos mesmos acerca das suas impressões quanto ao uso do protótipo. Este questionário consistiu de seis afirmações estruturadas por meio de escalas do tipo Likert, variando de 1 (discordo totalmente) a 5 (concordo totalmente). Ele foi aplicado para um total de vinte e nove alunos.

\section{Resultados}

O primeiro questionário $(\mathrm{n}=25)$ teve como objetivo verificar a opinião dos alunos em relação às dificuldades por eles observadas quanto ao aprendizado dos principais conteúdos da disciplina, bem como a possibilidade de utilização de recursos tecnológicos para este fim.

A primeira pergunta feita foi "Você acha a bioquímica uma disciplina difícil?" Em relação à dificuldade em Bioquímica Estrutural, 60\% $(n=15)$ consideraram a Bioquímica Estrutural uma disciplina difícil. Destes, 26,60\% atribuem a dificuldade à complexidade do assunto, $20 \%$ à dificuldade em entendimento de química, $20 \%$ não especificaram, $13,3 \%$ ao conteúdo extenso, $13,3 \%$ ao detalhamento excessivo e $6,6 \%$ à complexidade do livro (Figura 1). Com isso, constata-se que a maioria dos alunos considera a bioquímica uma disciplina difícil, destacando-se principalmente o excesso e dificuldade de conceitos. A dificuldade em química dos alunos de bioquímica reforça o que é visto na literatura e pode ser explicada pelo contato ainda prematuro com essa área, visto que a disciplina é ofertada geralmente no primeiro ano dos cursos, associado a um ensino médio deficiente (ZENI, 2010; ANDRADE et al., 2017).

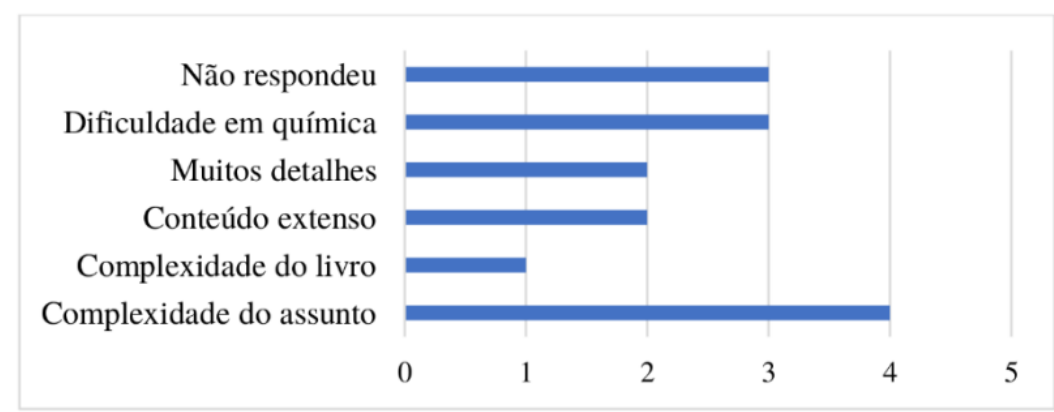

\section{Figura 1. Número de alunos relacionados às principais dificuldades encontradas.}

A segunda questão pedia ao aluno para enumerar, em ordem decrescente de relevância, as suas maiores dificuldades dentro da disciplina. Dentre os itens enumerados, os alunos consideram as maiores dificuldades no conteúdo de Bioquímica Estrutural citar as funções biológicas das biomoléculas (31\%), seguido por "identificar interações intermoleculares" (27\%) e a visualização espacial das biomoléculas (18\%) (Figura 2). As duas maiores dificuldades são assuntos ministrados no ensino médio e revisados na 
graduação e podem ser explicadas por um ensino médio deficiente. A terceira maior dificuldade pode ser explicada pelo menor contato com a abstração de biomoléculas, visto que não é praticado durante o período escolar e é necessário alto grau de exigência dessa habilidade para a compreensão dos processos bioquímicos.

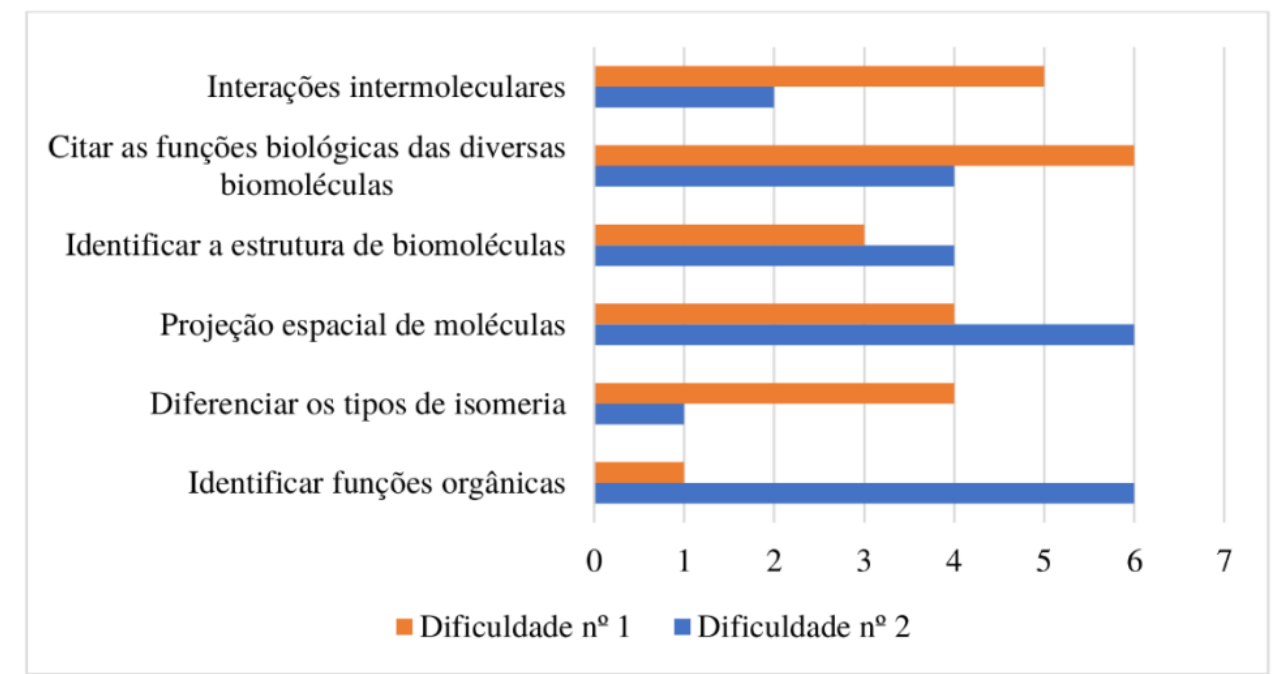

Figura 2. Número de alunos relacionados aos tópicos de maior dificuldade dentro da disciplina de Bioquímica Estrutural.

$\mathrm{Na}$ terceira pergunta, foi questionado se os alunos utilizavam algum dispositivo eletrônico - celular, tablet, computador, etc - para estudar. Quanto à utilização de dispositivos eletrônicos para o estudo, $92 \%(n=23)$ responderam que os usavam, dentro os quais $70 \%$ utilizam o celular e $30 \%$ o computador. Outros $4 \%$ responderam que não utilizam nenhum aparelho eletrônico e outros $4 \%$ não responderam à pergunta. Esse resultado corrobora com o estudo de DINIZ, 2013, que mostra que a maioria dos estudantes universitários utiliza pelo menos um dispositivo eletrônico para estudar, de modo a facilitar a pesquisa, expandir as informações disponíveis e melhorar o desempenho acadêmico.

$\mathrm{Na}$ quarta questão, foi perguntado se os alunos utilizavam algum aplicativo para estudar Bioquímica Estrutural e se positivo, para citar alguns deles. A maioria dos alunos $(96 \%, n=24)$ responderam que não utilizam, enquanto $4 \%$ responderam que utilizavam. Alguns alunos que responderam "sim", confundiram os termos aplicativo e website. Esse resultado provavelmente acontece pela escassez de programas voltados para o tema, uma vez que a maioria utiliza celular ou computador para estudar e poucos utilizam algum aplicativo.

Na quinta questão os alunos deveriam responder à pergunta: "Você acha que a criação de aplicativos voltados à bioquímica poderia ajudar na compreensão dos assuntos ministrados durante a disciplina?" e, em caso de resposta afirmativa foi solicitado que comentasse alguns recursos ou utilidades que esses aplicativos deveriam conter (Figura 3). Todos os alunos entrevistados responderam "sim" para a pergunta em questão, sugerindo que aplicativos deveriam possuir ferramentas para melhor fixação do assunto, acesso a informações textuais e a apresentação de estruturas tridimensionais. 


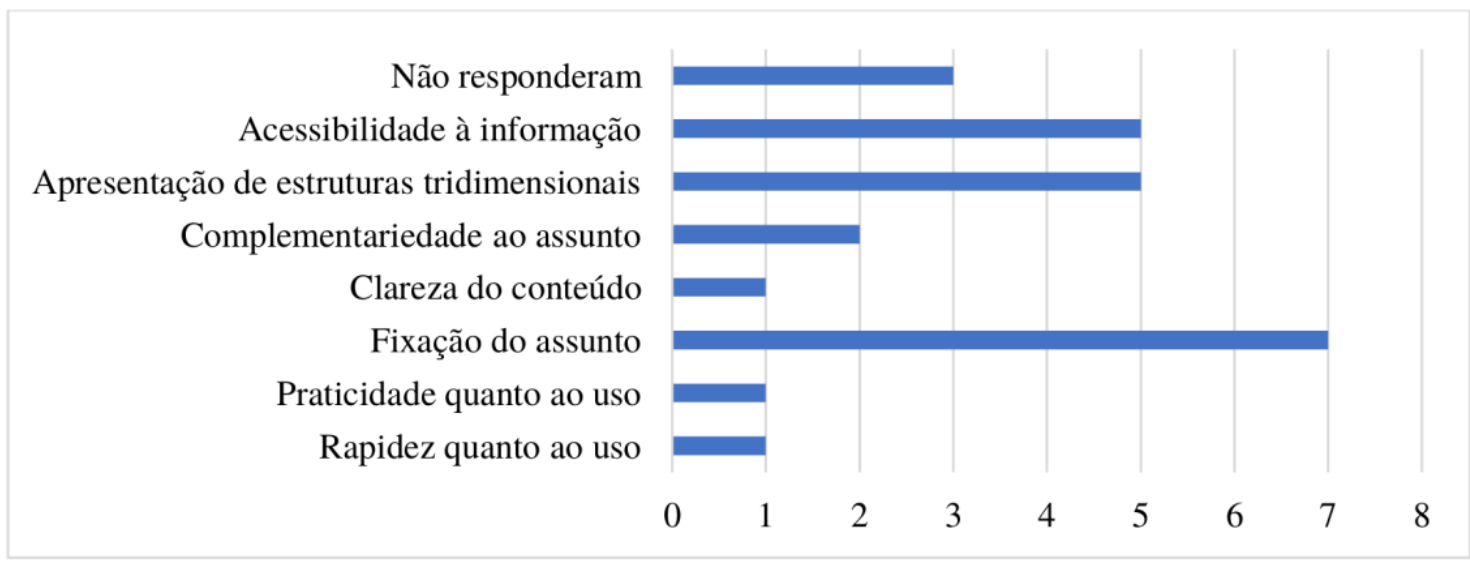

Figura 3. Número de alunos relacionados aos principais recursos de um aplicativo desenvolvido para a disciplina.

$\mathrm{Na}$ última questão do primeiro questionário, foi perguntado, baseado nas respostas da quinta questão, quais conteúdos (textos, vídeos, animações, etc) ele conteria e como seria sua estrutura (quiz, mapas mentais, etc). Dentre as propostas, os alunos responderam que um aplicativo para bioquímica estrutural deveria conter resumos $(64 \%$, $\mathrm{n}=16)$, questões comentadas $(16 \%, \mathrm{n}=4)$, animações $(12 \%, \mathrm{n}=3)$ e/ou mapas mentais $(4 \%, n=1)($ Figura 4$)$.

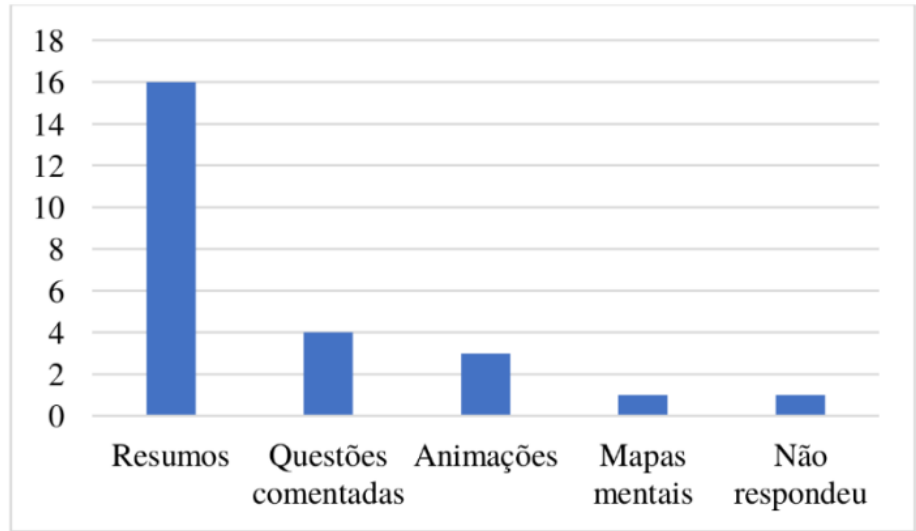

Figura 4. Principais conteúdos que devem estar presentes no aplicativo relacionado ao número de alunos.

Os resultados da primeira etapa da pesquisa permitiram identificar que os alunos possuem dispositivos tecnológicos $(96 \%)$ e que, além de outras possibilidades, gostariam de poder identificar e compreender melhor aspectos estruturais e espaciais relacionados ao conteúdo da disciplina. Entretanto, os alunos não conseguiram expressar como gostariam que isso ocorresse, senão por informações textuais (resumos, questões comentadas e mapas mentais) e animações, evidenciando a forma bidimensional presente no aprendizado tradicional baseado em aula expositiva em quadro ou livros.

Uma vez que as questões espaciais foram identificadas com uma das principais dificuldades dos alunos (questão 2, dificuldade número 1), iniciou-se o desenvolvimento de um protótipo de visualização espacial baseado no uso de realidade aumentada. Neste protótipo, optou-se por incrementar a informação textual e gráfica dos livros e materiais impressos com o auxílio de um aplicativo para dispositivos móveis. Por meio do 
protótipo, os alunos puderam experimentar a manipulação interativa de uma molécula em 3D, sobreposta automaticamente ao desenho bidimensional desta molécula (Figura $5)$.

A partir dos dados obtidos com o primeiro questionário e a apresentação do protótipo para a turma, um segundo questionário foi feito, com o objetivo de verificar a utilidade do aplicativo para a disciplina, bem como a possibilidade de visualização e interação com o modelo apresentado. Com os resultados obtidos a partir do segundo questionário $(\mathrm{n}=29)$, foi feita uma tabela (Tabela 1) relacionando cada afirmação com o grau de concordância dos alunos, em que quanto maior a proximidade de 5, maior o grau de concordância.
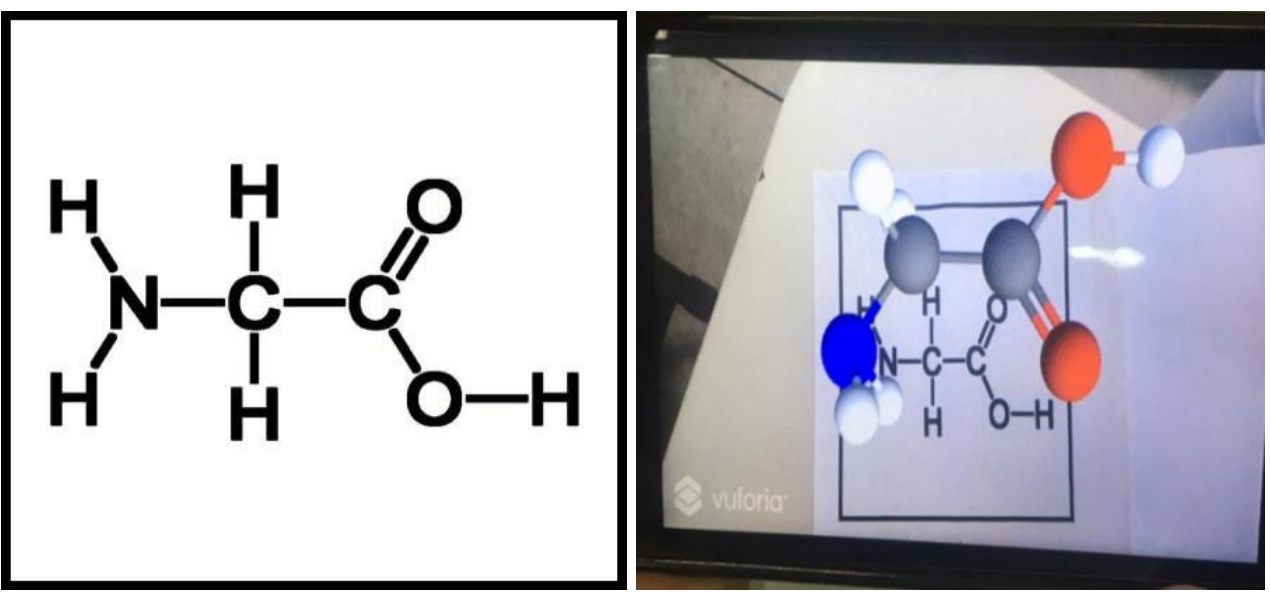

Figura 5. Representação estrutural bidimensional da molécula da glicina (esquerda) e protótipo de realidade aumentada em execução com a sobreposição da molécula tridimensional (direita).

Tabela 1. Respostas do segundo questionário.

\begin{tabular}{l|c}
\hline \multicolumn{1}{c|}{ Afirmação } & Média \\
\hline Eu usaria um aplicativo baseado nesse "protótipo" & $\mathbf{4 , 9 0}$ \\
\hline $\begin{array}{l}\text { O processo educacional de bioquímica seria mais produtivo com a } \\
\text { utilização deste aplicativo. }\end{array}$ & $\mathbf{4 , 8 3}$ \\
\hline $\begin{array}{l}\text { O processo educacional de bioquímica seria mais interessante com a } \\
\text { utilização deste aplicativo. }\end{array}$ & $\mathbf{4 , 8 7}$ \\
\hline Este aplicativo poderia ser útil para o ensino de Bioquímica Estrutural. & $\mathbf{4 , 9 3}$ \\
\hline Eu pagaria um valor simbólico para utilizar o aplicativo. & $\mathbf{4 , 2 0}$ \\
\hline $\begin{array}{l}\text { Este aplicativo seria mais interessante se acompanhado por conteúdo } \\
\text { textual. }\end{array}$ & $\mathbf{4 , 7 6}$ \\
\hline
\end{tabular}

A escala tipo Likert é constituída por itens em que o respondente será avaliado 
para manifestar a sua opinião de acordo ou desacordo com a afirmação feita e o grau de intensidade das respostas (PASQUALI, 2011). A partir disso, verificou-se que a maioria dos alunos concorda com afirmações "Este aplicativo poderia ser útil para o ensino de Bioquímica Estrutural", "Eu usaria um aplicativo baseado neste protótipo" e "O processo educacional de bioquímica seria mais interessante com a utilização deste aplicativo".

\section{Discussão e Perspectivas}

Com base nas respostas obtidas com os questionários aplicados, conclui-se que os alunos têm a percepção de que a Bioquímica Estrutural é uma disciplina cuja dificuldade está relacionada à complexidade do assunto, o qual depende em parte do conhecimento de aspectos básicos da química, a saber: identificação das principais funções químicas e compreensão das estruturas espaciais de moléculas. Além disso, a maioria dos alunos afirmou que utiliza de dispositivos eletrônicos em seus estudos e que a criação de aplicativos que possibilitem a interação com a estrutura tridimensional de moléculas seria uma estratégia importante no processo ensino-aprendizagem do componente curricular Bioquímica Estrutural. Com a criação do protótipo de um aplicativo voltado para o ensino da disciplina, os alunos poderão se beneficiar com a praticidade da tecnologia por via de smartphone, bem como facilitar o acesso ao conteúdo, com um papel complementar no aprendizado da disciplina de forma a tornar seu conteúdo mais didático e tecnológico.

Com base nas respostas obtidas com os questionários aplicados, conclui-se que os alunos têm a percepção de que a Bioquímica Estrutural é uma disciplina cuja dificuldade está relacionada à complexidade do assunto, o qual depende em parte do conhecimento de aspectos básicos da química, a saber: identificação das principais funções químicas e compreensão das estruturas espaciais de moléculas. Além disso, a maioria dos alunos afirmou que utiliza de dispositivos eletrônicos em seus estudos e que a criação de aplicativos que possibilitem a interação com a estrutura tridimensional de moléculas seria uma estratégia importante no processo ensino-aprendizagem do componente curricular Bioquímica Estrutural. Com a criação do protótipo de um aplicativo voltado para o ensino da disciplina, os alunos poderão se beneficiar com a praticidade da tecnologia por via de smartphone, bem como facilitar o acesso ao conteúdo, com um papel complementar no aprendizado da disciplina de forma a tornar seu conteúdo mais didático e tecnológico.

Uma vez que uma das maiores dificuldades apontadas pelos alunos foi a visualização espacial de biomoléculas, um recurso que utilize a realidade aumentada poderia suprir essa necessidade. Além disso, essa tecnologia permitiria maior interação, com a possibilidade de rotação, visualização de funções orgânicas, modificações no tamanho das biomoléculas e identificação de padrões moleculares.

Dentre os conteúdos ministrados na disciplina, destaca-se o tópico de aminoácidos para a realização de um aplicativo baseado na realidade aumentada. Essas biomoléculas possuem uma estrutura conservada dentro as diversas moléculas que compõem o grupo e uma porção distinta, onde há mudanças espaciais relevantes, relevantes para a compreensão da atividade biológica e de difícil visualização. Além disso, em sua estrutura, existem diferentes tipos de funções orgânicas, o que permitiria o estudo e a diferenciação destes grupos químicos pelos alunos, reparando uma das maiores dificuldades apontadas.

Com o objetivo de suprir a demanda da disciplina tanto na dificuldade de assimilação do conteúdo quanto na escassez de recursos tecnológicos para o ensino, está 
sendo desenvolvido um aplicativo de realidade aumentada baseado na estrutura tridimensional dos aminoácidos, associados a um conteúdo textual complementar. Uma vez que o protótipo foi apenas para verificar a aceitação da tecnologia de RA pelos alunos, os demais elementos identificados na $2^{\mathrm{a}}$ questão do primeiro questionário estão sendo considerados no processo de desenvolvimento do aplicativo.

Assim sendo, esse programa terá como objetivo ampliaras ferramentas de ensino da Bioquímica Estrutural por meio do uso da tecnologia, que é o principal recurso utilizado nos tempos atuais pelos alunos na busca de informações de ensino de disciplinas. Com isso, o aplicativo será utilizado como um recurso complementar e didático, sendo uma extensão do ensino em sala de aula e promovendo uma interação com a disciplina fora das paredes da Universidade.

\section{Referências}

Alcântara, N.; Vieira, A. (2015) "Elaboração e utilização de um aplicativo como ferramenta no ensino de Bioquímica: carboidratos, lipídios, proteínas e ácidos nucleicos". Revista de Ensino de Bioquímica 13(3): 54-72.

Andrade, R.S.B.; Silva, A.F.S.; Zierer, M.S. (2017) "Avaliação das dificuldades de aprendizado em Bioquímica dos discentes da Universidade Federal do Piauí". Revista de Ensino de Bioquímica 15(1): 24-39. Sociedade Brasileira de Bioquimica e Biologia Molecular - SBBq. DOI:10.16923/reb.v15i1.690.

Diniz, C. N.; Ol, G.O. (2006) "O uso da tecnologia da informação entre universitários" In: $7^{\circ}$ ENANCIB - Encontro Nacional de Pesquisa em Ciência da Informação.

Garzón, J. C. V; Magrini, M. L; Costa, C; Galembeck, E. (2014) "Metodologias inovadoras: Realidade aumentada no ensino de vias metabólicas". Revista de Ensino de Bioquímica, 12(2): 128-143.

Mitre, S. et al. (2008) "Metodologias ativas de ensino-aprendizagem na formação profissional em saúde: debates atuais". Ciência Saúde Coletiva 13(2): 2133-44.

Nelson, D. L.; Cox, M. M. (2014), Princípios de Bioquímica de Lehninger, Artmed, $6{ }^{\text {a }}$ Edição.

Lima, E. F. (2017) “O Uso das TICs e da Pesquisa como Recursos Pedagógicos no Ensino de Bioquímica para o Curso de Licenciatura em Química”. Revista de Graduação USP, 2(2): 115-120.

Pasquali, L. (2011), Psicometria: Teoria dos testes na psicologia e educação, Vozes, $4^{\mathrm{a}}$ Edição.

Rezende, F. (2000) “As Novas Tecnologias na Prática Pedagógica sob A Perspectiva Construtivista". Revista Ensaio 2(1):70-87.

Souza, F. N. (2006) "Perguntas na aprendizagem de Química no Ensino Superior". [Dissertação]. Universidade de Aveiro, Aveiro, 2006.

Tori, R.; Hounsell, M.S. (2018), Introdução a Realidade Virtual e Aumentada, $1^{\mathrm{a}}$ edição.

Yokaichiya, D. K.; Galembeck, E.; Torres, B. B. (2004) "O que alunos de diferentes cursos procuram em disciplinas extracurriculares de bioquímica”. Revista Brasileira de Ensino de Bioquímica e Biologia Molecular 1:E1-E8. 
Zeni, A.L.B. (2010) "Conhecimento prévio para a disciplina de bioquímica em cursos da área da saúde da Universidade Regional de Blumenau-SC". Revista de Ensino de Bioquímica 2(1):B1-B14. 